\title{
Getreide-Erntebeginn und Frühsommertemperaturen im schweizerischen Mittelland seit dem 17. Jahrhundert ${ }^{\star}$
}

\section{Einleitung und Problemstellung}

Die intensive klimageschichtliche Forschung der letzten Jahre, von einer zunehmenden Sorge um mögliche zukünftige natürliche oder anthropogene Klimamodifikationen getragen, ist in zwei Richtungen vorangetrieben worden. Einmal ist die Zeitachse um Jahrmillionen nach rückwärts verlängert worden, und die Frage nach dem Klima ganzer geologischer Epochen ist unter dem Gesichtspunkt neuer wissenschaftlicher Erkenntnisse (Plattentektonik!) und mit Hilfe neuer Methoden (Analyse von Tiefseebohrkernen) neu aufgerollt worden; (MARGOLIS und KENNETH, 1971, SPJELDNESS, 1978). Andererseits ist das zeitliche Auflösungsvermögen des bestehenden klimageschichtlichen Records in bisher ungeahntem Maße verfeinert worden: mit Hilfe der Sauerstoffisotopenmethode $\left(16_{0} / 18_{0}\right)$ ist es beispielsweise möglich, die Temperaturentwicklung über die letzten 120000 Jahre hinweg zu verfolgen (DANSGAARD et al, 1969), während die neuentwickelte Radio-Dendrochronologie eine Rekonstruktion der Spätsommertemperaturen (Juli-September) für die letzten 5-7 Jahrhunderte erlaubt (RÖTHLISBERGER, 1976; SCHWEINGRUBER, in Vorb). Ein noch besseres zeitliches Auflösungsvermögen in der Größenordnung von Tagen und Stunden bieten ausführliche Beschreibungen des Witterungsverlaufs. Sie lassen sich, wenn sie lückenlos für mehrere Jahre oder Jahrzehnte vorliegen, quantifizieren und zu modernen Klimadaten in Beziehung setzen (FLOHN, 1949; PFISTER, 1978a).

Daneben finden wir auch in historischen Dokumenten Proxy-Daten, also Informationsträger, die, wie die Eisbohrkerne der Physiker oder die Pollenprofile der Botaniker, als indirekte Indikatoren von Klimavariablen verwendet werden können. Dazu gehören einmal die Angaben über die Blüh- und Reifedaten von Kulturpflanzen, die sich in alten Chroniken finden. Die Chronisten haben die Vegetationsentwicklung mit Vorliebe in sehr frühen und sehr späten Jahren festgehalten, um damit einen objektiveren Maßstab für die Wärme und Kälte zu gewinnen. Solche Einzelbeobachtungen können wertvolle Anhaltspunkte für die Größenordnung von Temperatur-Anomalien liefern. In manchen Gegenden wurde früher der Zeitpunkt der Weinlese von der Lokalbehörde festgelegt. Bevor diese die Reife der Trauben begutachtet und durch einen

\footnotetext{
* Diese Arbeit ist im Rahmen eines vom Schweiz. Nationalfonds unterstützten Forschungsprogramms entstanden.
}

förmlichen Beschluß die Lese freigegeben hatte, durften die Weinberge nicht betreten werden. Die Akten, in welchen diese Beschlüsse festgehalten sind, haben sich da und dort erhalten, so daß es unter günstigen Umständen möglich wird, die Fluktuationen des Weinlesebeginns über Jahrhunderte hinweg zu verfolgen. In der Schweiz reicht die längste Reihe dieser Art ins späte 15. Jahrhundert zurück (DUFOUR, 1870). LE ROY LADURIE (1978) verglich den über 102 französische, westdeutsche und westschweizerische Serien gemittelten Lesebeginn von Jahr zu Jahr mit den Durchschnittstemperaturen der Monate April-September in Paris und erhielt eine mit einem Korrelationskoeffizienten von 0.86 erstaunlich gute Übereinstimmung. Allerdings kann der Fehler bei der Schätzung der Temperatur in einzelnen Sommern recht groß werden (DE VRIES, 1977). Ökologische und anthropogene Faktoren sind als Ursache zu vermuten:

- einmal sind für die Entwicklung der Trauben vor allem die Temperaturen oberhalb eines bestimmten Schwellenwerts und nicht Durchschnittswerte aus mehreren täglichen Messungen maßgegend, wie sie den meteorologischen Daten zugrundeliegen (PEYER, KOBLET, 1966). Außerdem scheinen die Frühsommertemperaturen (Mai, Juni) für den Zeitpunkt der Lese wesentlich stärker ins Gewicht zu fallen als August und September (PFISTER, 1978b), weshalb der Aussagewert der Lesedaten als Indikator für die Durchschnittstemperatur der Monate April bis September fraglich erscheint. Schließlich konnte TRENKLE (1969) starke sortenspezifische Unterschiede zwischen verschiedenen Traubensorten nachweisen.

- wesentlich schwerer fallen die anthropogenen Faktoren ins Gewicht: eine frühe Lese ist nicht in jedem Falle das Produkt eines warmen, sonnigen Sommers. Noch heute müssen die Trauben bei fortgeschrittener Fäulnis oder nach starken Frösten gelesen werden, ehe sie vollständig reif sind. Zahlreiche Kommentare von Witterungschronisten machen deutlich, daß dies auch früher der Fall war, und zwar, entsprechend dem ungünstigeren Klima des "Little Ice Age», häufiger als im 19. und 20. Jahrhundert. Zudem sind verschiedene Fälle bekannt, wo die Trauben überhaupt nicht zur Reife kamen, so daß auch ein sehr spätes Lese-

Dr. Christian Pfister, Geographisches Institut der Universität Bern, Hallerstraße 12, 3012 Bern. 
datum den wahren thermischen Bedingungen nicht immer entspricht. Im weiteren weist LE ROY LADURIE (1971) darauf hin, daß man in manchen Weinbaugebieten im Verlaufe des 18. und 19. Jahrhunderts von einer quantitätsorientierten zu einer qualitätsorientierten Produktionsweise überging, was dazu führte, daß man die Trauben länger an den Stöcken hängen ließ. Für eine detaillierte Charakterisierung der Sommertemperaturen während des sogenannten «Little Ice Age», namentlich für die Beurteilung von Gletscherschwankungen, bieten die Weinlesedaten allein jedenfalls keine tragfähige Basis. Es ist nötig, sie durch weitere Typen von Proxy-Daten zu ergänzen.

Getreide-Zehnterträge, also Indikatoren für den Umfang der Ernte, sind von STAUFFER und LÜTHI (1974) auf ihre Eignung als Klimaindikatoren überprüft worden. Eine 270 Jahre umfassende Serie vonErträgen mehrerer aargauischer Bezirke wurde mit einem Bohrkern aus dem grönländischen Inlandeis verglichen, bei welchem der wechselnde Gehalt an Sauerstoffisotopen $\left(16_{0} / 18_{0}\right)$ Hinweise auf die mittlere Jahrestemperatur gestattet. Anhand einer Spektralanalyse ergab sich eine überraschende signifikante Korrelation zwischen dem Isotopenverhältnis im Eis und den Fluktuationen der Zehntenerträge, woraus die beiden Autoren den Schluß ziehen, «daß gewisse Schwankungen der Ernteerträge durch weiträumig wirksame Klimaschwankungen bedingt seien». Die Korrelation ist jedoch sogar mit den Jahrestemperaturen einer nahegelegenen Station wie Genf (ab 1753) mit 0,14 enttäuschend klein und nicht signifikant, was angesichts der Komplexität der ertragsbestimmenden Witterungsfaktoren beim Getreide (KOBLET 1965, PFISTER 1975; JEANNERET 1978) nicht erstaunt. Wesentlich günstiger als Getreide-Erträge sind Angaben über den Zeitpunkt der Ernte, die, wie eine Analyse langjähriger phänologischer Reihen aus dem Kanton Schaffhausen (1876-1953) ergeben hat, als Indikatoren der Frühjahrs- und Frühsommertemperaturen verwendet werden können (PFISTER 1978b). Die aus der Zeit vor 1750 vorliegenden direkten Erntebeobachtungen (Tab. 1) sind entweder zeitlich vollständig isoliert, oder dann ist die Überlappung mit der 1755 beginnenden Basler Temperaturreihe (BIDER, SCHÜEPP, v. RUDLOFF, 1959) für die Berechnung eines brauchbaren Schätzmodells zu kurz. Proxy-Daten des Erntebeginns, die sogenannten Zehntsteigerungsdaten, bringen diesbezüglich günstigere Voraussetzungen mit: sie liegen in Form langer, sich gegenseitig überlappender Serien vor, von welchen einige bis ins frühe 17. Jahrhundert zurückreichen, und zugleich den Zeitraum von der Mitte des 18. bis ins frühe 19. Jahrhundert umfassen, der durch Temperaturmessungen abgedeckt ist.

Die vorliegende Arbeit setzt sich ein dreifaches Ziel. Zuerst geht es darum, den Bezug zwischen Zehntsteigerungsdaten und Erntebeginn näher zu untersuchen und sie in einen räumlich-ökologischen Bezugsrahmen . zu stellen. In einem zweiten Schritt soll aus den vorhandenen direkten und indirekten Daten eine möglichst homogene Säkularreihe aufgebaut werden, welche die Fluktuationen des Erntebeginns zur Darstellung bringt. Schließlich soll abgeklärt werden, inwiefern diese Reihe als Schätzgröße von Monatstemperaturen oder Temperatursummen des Zeitraums von April bis Juli geeignet ist.

\section{Zehntsteigerungsdatum und Datum des Ernte- beginns}

Streng genommen ist nur der Reifezustand des Getreides eine Funktion des Witterungsverlaufs und der Temperaturverhältnisse. Der Zeitpunkt der Ernte war und ist auch von nichtklimatischen Randbedingungen abhängig. Im Ancien Regime war es die Rücksichtnahme auf die koordinierte Bewirtschaftung der Felder unter dem System des Flurzwangs und die Institution des Zehntens; unter den heutigen Bedingungen ist es die Verfügbarkeit der Mähdrescher. Einzig zwischen dem frühen 19. Jahrhundert und der technologischen Revolution der Landwirtschaft in der zweiten Hälfte unseres Jahrhunderts war der Bauer frei zu entscheiden, wann er mit der Ernte beginnen wollte. Wenn wir Zehntsteigerungsdaten als Klima-Indikatoren verwenden wollen, gilt es vorerst zu prüfen, inwieweit der Zehntbezug und die Bewirtschaftungsweise im Dreizelgensystem unter dem Ancien Regime auf die ökologischen Bedingungen abgestimmt worden sind.

Der Zehnte ist seiner Rechtsnatur nach eine kirchliche Naturalabgabe, die von allen Erträgnissen eines damit belasteten Landstücks geschuldet wurde. Den bedeutendsten Teil machten im «Kornland» die Getreidezehnten aus, die, wie der Name sagt, in den meisten Gegenden ein Zehntel der Ernte betrugen. In den protestantischen Kantonen gingen die kirchlichen Zehnten nach Aufhebung der Klöster in der Reformation in den Besitz der Obrigkeiten über, für welche sie die wichtigsten Einnahmequellen blieben, bis die Loskaufsgesetze des frühen 19. Jahrhunderts es den Bauern ermöglichten, sich von der verhaßten Abgabe zu befreien. Da die Zehntherren mit den Verhältnissen in den einzelnen Dörfern zu wenig vertraut waren und über keine Beamtenschaft verfügten, welche den Bezug hätte überwachen können, war es üblich, die Zehnten unter den wohlhabenden Bauern eines Dorfes zu versteigern. Der Zehntbesteher, welchem ein Zehnten um eine bestimmte Menge zugeschlagen wurde, verpflichtete sich, das Getreide in eigener Regie einzusammeln und zum vereinbarten Zeitpunkt sauber gedroschen in die Zehntenscheuer zu liefern. Um sich vor der Versteigerung ein Bild über den Zustand des Getreides und die zu erwartenden Erträge zu beschaffen, beauftragte der Zehntherr Vertrauensleute damit, «die Zehenden zu beschouen». Bei diesen Zehntschätzern handelte es sich um Mitarbeiter des Landvogts oder wohlhabende Bauern, die vereidigt und entschädigt wurden. Ein bis acht Tage vor der Versteigerung besichtigten sie die Flur und schlossen auf Grund von Aussehen, Dichte und 
Fig. 1: Zehntverleihungsdaten und Erntebeginn im Zeitraum 1717-1767

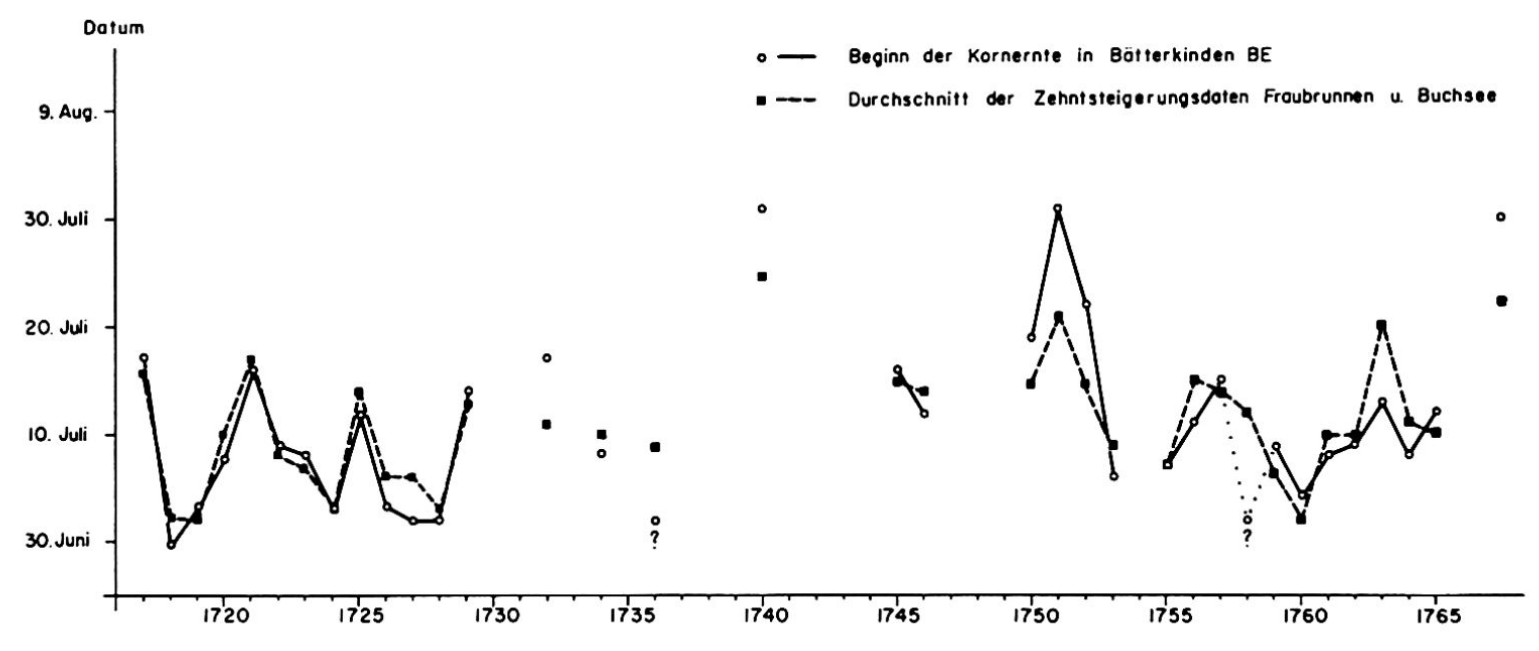

Ouelle: Kornernte-Daten: WOLF, 1853

Unkrautbefall der in einer Zelg angebauten Getreidesorten auf den zu erwartenden Ertrag. Diese sogenannte Schatzung durfte nicht unterboten werden. Das Datum der Versteigerung wurde zugleich mit der Schatzung anberaumt und in den betroffenen Kirchgemeinden von der Kanzel verkündet. Im alten Bern fand der Versteigerungsakt in Anwesenheit des Landvogts statt, war mit einem üppigen Mahl verbunden und ging oft in ein Volksfest über. Der Beginn der Ernte mußte unter dem geltenden Flurzwang von allen Benützern einer Zelg gemeinsam festgestellt werden. Vorgängig waren die Felder an vielen Orten wie die Weinberge «gebannt», das heißt streng gesperrt, so daß nicht einmal die Grundbesitzer auf ihre Parzellen gehen durften. Nach der Freigabe der Ernte, die in der Regel unmittelbar auf die Zehntsteigerung folgte, hatte der Bauer die 10., 20., 30. Garbe aus der Reihe zu stellen, oder sie sonst irgendwie deutlich als Zehntgarbe zu kennzeichnen (GMÜR 1954). Die abzuliefernden Getreidemengen und die Namen der Zehntbesteher wurden in die Zehntrödel eingetragen. $\mathrm{Ob}$ diese datiert sind und damit zu Quellen für die Klimaforschung werden, hing von der Laune des Schreibers und der örtlichen Tradition ab. Sobald das Zehntgetreide abgeliefert und in der Amtsrechnung demselben Gebiet (Bätterkinden BE), wird deutlich, $\mathrm{da} \beta$ die beiden Kurven im allgemeinen übereinstimmen (Fig. 1). In den meisten Fällen folgt der Erntebeginn unmittelbar auf die Verleihung. Bemerkenswert ist, daß man in sehr späten Jahren $(1740,1751,1763$, 1767) nach der Versteigerung mit dem Schnitt des Geverbucht war, verloren die Zehntrödel ihren dokumentarischen Wert. Dies mag ein Grund dafür sein, weshalb nur verhältnismäßig wenige Serien erhalten sind. Vergleichen wir die gemittelten Zehntsteigerungsdaten der Landvogteien Fraubrunnen und (München)-Buchsee mit Aufzeichnungen über den Erntebeginn aus treides länger zuwartete als üblich. Dies deutet darauf hin, daß stark negative Temperaturabweichungen durch die Steigerundsdaten gedämpft werden. Auch die Daten des frühen 17. Jahrhunderts können als hervorragende Indikatoren betrachtet werden: der Korrelationskoeffizient der beiden Schaffhauser Reihen (vgl. Kap. 3) mit den unmittelbaren Beobachtungen des Erntebeginns in Winterthur durch Anton Künzli (vgl. Tab. 1) beträgt 0.85 (S.0.00001). Daß sich der Zeitpunkt der Schatzung in erster Linie nach dem Reifezustand des Getreides richtete, geht aus mehreren Schreiben bernischer Landvögte an den Seckelmeister hervor. So lesen wir in einem Brief des Bipper Landvogts vom 8. August 1970:

«Es ist zu wissen, daß die Zehnden im Berg als Rumisberg Farneren und Wolfisberg (in einer Höhenlage von 600-800 m) wegen später Jahrszeit und da noch ungewiß was solche abtragen, dißmahl noch nicht haben hingeliehen werden können» (STAB 1).

Wenn die Verleihung bei einem zu weit fortgeschrittenen Reifezustand erfolgte, bestand die Gefahr von Verlusten. Im Jahre 1806 wurde das schlechte Ergebnis der Versteigerung im Amt Wangen damit begründet, da $ß$ «das Korn an vielen Orten überreiff und sehr mürbe ist, daher durch das Abfallen (der Körner) vieles verloren geht» (STAB 2). Aus wirtschaftlichen Erwägungen suchte man auch die Zeitspanne zwischen Schatzung, Hinleihung und Erntebeginn so weit wie möglich zu verkürzen. Während dieser Zeit blieben die Felder weiterhin dem Risiko von Hagelschlag, Nässeschaden und Parasitenbefall ausgesetzt. Eine Neuschatzung war mit zusätzlichen Kosten verbunden (STAB 3), die, wenn eine solche nach der Versteigerung nötig wurde, der Zehntbesitzer zu tragen hatte. Eine Anpassung des Schatzungs- und Versteigerungsdatums an die ökologischen Bedingungen hat sich in manchen Gebieten 


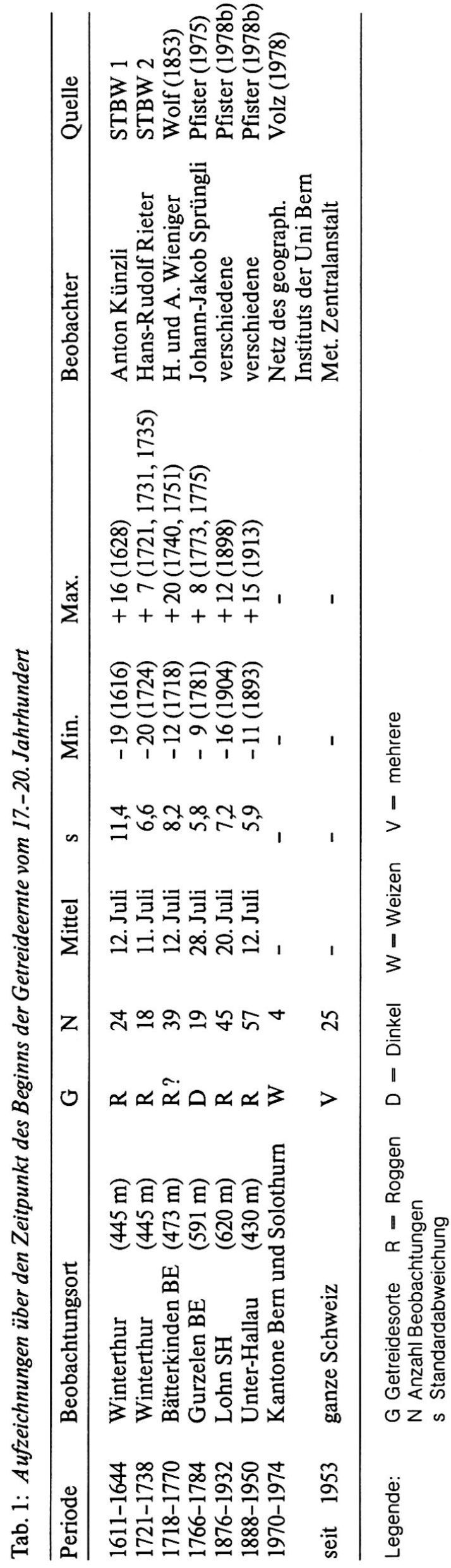

allerdings erst im Verlaufe der Zeit eingependelt. So wurden die Zehnten im zürcherischen Illnau im späten 16. Jahrhundert bevorzugt an einem Sonntag, meistens am Sonntag nach Johannis (24. Juni/4. Juli) verliehen, und zwar auch in Jahren, welche nach übereinstimmender Aussage vieler Chronisten und Beobachter große zeitliche Unterschiede in der Vegetationsentwicklung aufweisen. Kurz vor der Jahrhundertwende erfolgte die Verschiebung auf den Dienstag, der daraufhin für einige Jahrzehnte der bevorzugte Tag für Zehntsteigerungen blieb. Im zürcherischen Andelfingen wiederum findet sich in demselben Zeitraum eine reiche Variationsbreite an Wochentagen, was darauf schließen läßt, daß man sich dort nicht auf einen bestimmten Tag festgelegt hatte. Es ist anzunehmen, daß solche anthropogene Abweichungen stochastisch verteilt sind und sich im Mittel einer größeren Anzahl von Serien ausgleichen.

\section{Die raum - zeitliche Gliederung der Daten}

Für die vorliegende Untersuchung sind Daten aus folgenden Quellen verwendet worden:

- Zehntrödel des säkularisierten Klosters Allerheiligen aus der Zeit von 1611 bis 1715. Leider umfaßt nur gerade eine einzige Serie - Illnau ZH - diesen Zeitraum. Kürzere Serien stammen aus dem süddeutschen Büßlingen, sowie aus Beggingen $\mathrm{SH}$ und Andelfingen ZH (STASH).

- Zehntrödel des Klosters Muri AG aus der Zeit zwischen 1658 und 1773. Für die Untersuchungen wurden die Steigerungsdaten der Flecken Bremgarten, Villmergen und Sursee, sowie der Dörfer Beinwil AG (Bez. Muri) und Neuenkirch LU verwendet (STAG).

- 35 Serien aus dem Gebiet des alten Bern. Die längsten stammen aus den Landvogteien (München)Buchsee, Erlach, Thorberg, Fraubrunnen und Wangen. Von 1741 an sind die Zehntrödel aus den deutschsprachigen Vogteien bis 1797 sozusagen lückenlos erhalten, doch sind leider nicht alle durchlaufend datiert. Nach der Zäsur während der Zeit der Helvetik, wo die Zehntpflicht zeitweise aufgehoben war, kehrte man zur vorrevolutionären Bezugspraxis zurück. Auch die Versteigerungsorte blieben im wesentlichen dieselben, nur daß die aargauischen Zehnten wegfielen und viele Zehntbezirke in den Grenzen der neugeschaffenen Amtsbezirke umgruppiert wurden (STAB 4).

Räumlich umfaßt die Untersuchung einen Streifen des Mittellandes, der im Norden durch den schaffhausischen Randen und die Thur, im Süden etwa durch eine Linie Guggisberg-Laupen-Erlach begrenzt ist.

Die Streuung des Besitzes vieler Landvogteien und Klöster über größere Teile des Mittellandes und mehrere Höhenstufen erzwang eine raum-zeitliche Staffelung der Steigerungsdaten. Einmal konnten den Bauern nicht zu lange Anmarschwege zugemutet werden; andererseits mußte der unterschiedliche Reifezustand des Getreides in verschiedenen Höhenlagen berücksichtigt werden. Exemplarisch läßt sich diese phänologisch be- 
Fig. 2: Mittlere Ernte- und Zehntverleihungsdaten in Abhängigkeit von der Höhenstufe

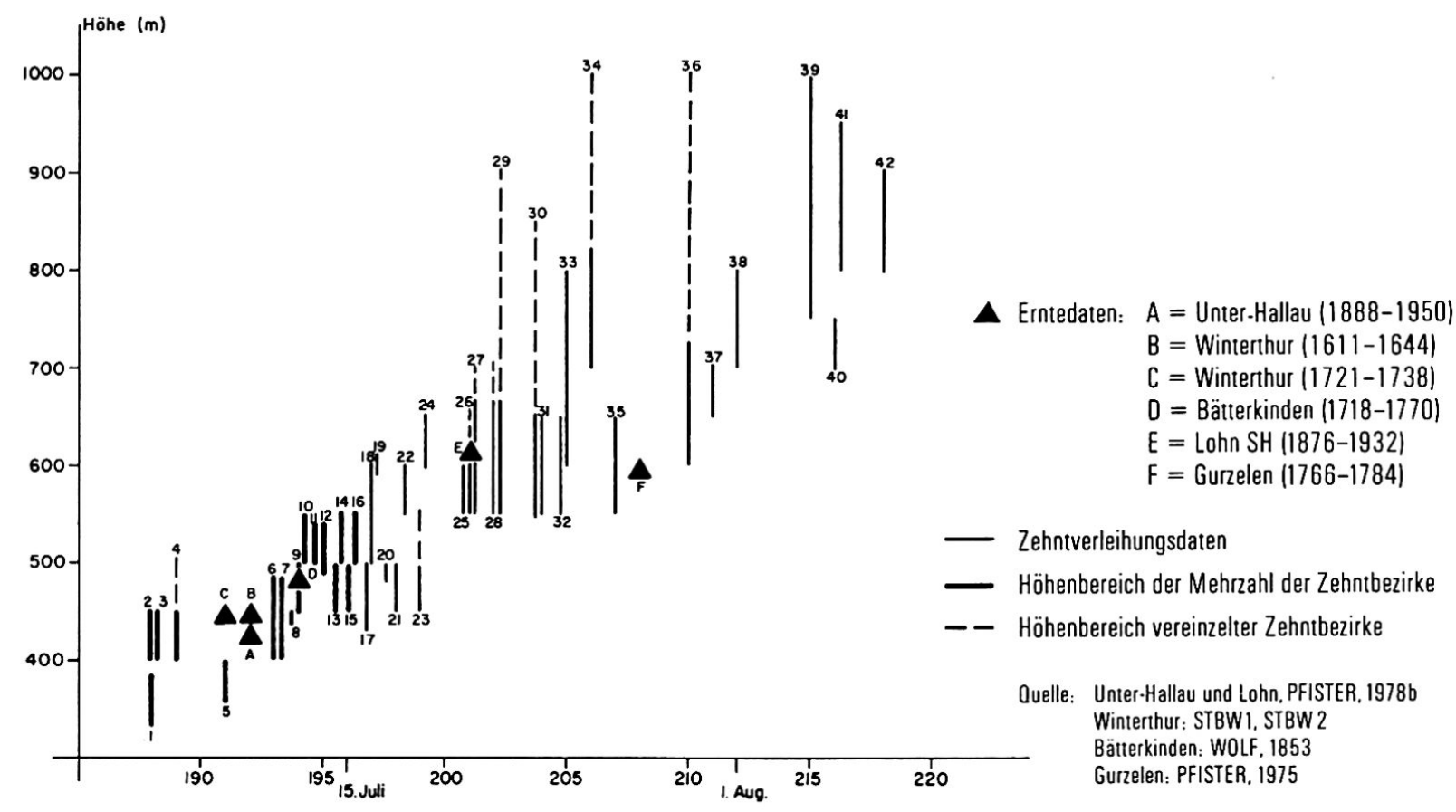

\section{Legende der Versteigerungsorte}

In Klammer: Name des Zehntbesitzers

$\mathrm{N}$ Anzahl Beobachtungen

Ldv. Landvogtei

KI. Kloster

Nr. Versteigerungsort/Zehntbesitzer N

1 Brugg (Ldv. Schenkenberg) 41

2 Brugg (Ldv. Königsfelden) 40

3 Villmergen (KI. Muri AG)

4 Bremgarten (KI. Muri AG)

5 Andelfingen (KI. Allerheiligen SH)

6 Lenzburg (Ldv. Lenzburg)

7 Gränichen (Schaffnerei Zofingen)

8 Aarberg (Ldv. Aarberg)

9 Oberwyl bei Büren a. A. (Stift Bern)

$\begin{array}{lll} & 10 & \text { Sursee (KI. Muri AG) } \\ & 11 & \text { Illnau (KI. Allerheiligen SH) } \\ & 12 & \text { Fraubrunnen (Ldv. Fraubrunnen) } \\ & 13 & \text { Landshut (Ldv. Landshut) } \\ & 14 & \text { Radelfingen b. Aarberg (Stift Bern) } \\ & 15 & \text { Büren (Ldv. Büren) } \\ \text { N } & 16 & \text { (München)-Buchsee (Ldv. Buchsee) } \\ & 17 & \text { Erlach (Ldv. Johannsen) } \\ 41 & 18 & \text { Frienisberg (Ldv. Frienisberg) } \\ 40 & 19 & \text { Beinwil AG (Bez. Muri; KI. Muri AG) } \\ 86 & 20 & \text { Koppigen/St. Niklaus (Ldv. Thorberg) } \\ 90 & 21 & \text { Nidau (Ldv. Nidau) } \\ 57 & 22 & \text { Bipp (Ldv. Bipp) } \\ 44 & 23 & \text { Herzogenbuchsee (Ldv. Wangen) } \\ 35 & 24 & \text { Seftigen (Oberamt Seftigen) } \\ 65 & 25 & \text { Neuenkirch LU (KI. Muri AG) } \\ 37 & 26 & \text { Bern (Stift Bern) }\end{array}$

$\begin{array}{rrlr}87 & 27 & \text { Bern (Schaffnerei St. Johannserhaus) } & 55 \\ 114 & 28 & \text { Bern (Schaffnerei Interlakenhaus) } & 35 \\ 118 & 29 & \text { Thorberg (Ldv. Thorberg) } & 63 \\ 76 & 30 & \text { Burgdorf (Ldv. Burgdorf) } & 21 \\ 38 & 31 & \text { Thun (Ldv. Thun) } & 65 \\ 74 & 32 & \text { Münsingen (Oberamt Konolfingen) } & 21 \\ 158 & 33 & \text { Rohrbach (Ldv. Wangen) } & 59 \\ 146 & 34 & \text { Walkringen (Ldv. Thorberg) } & 50 \\ 83 & 35 & \text { Köniz (Ldv. Köniz) } & 23 \\ 66 & 36 & \text { Trachselwald (Oberamt Trachselwald) } & 20 \\ 76 & 37 & \text { Schloßwyl (Oberamt Konolfingen) } & 18 \\ 76 & 38 & \text { Oberbalm (Stift Bern) } & 39 \\ 26 & 39 & \text { Schwarzenburg (Oberamt Schwarzenb.) } & 21 \\ 60 & 40 & \text { Langnau (Oberamt Signau) } & 10 \\ 23 & 41 & \text { Rüeggisberg (Stift Bern) } & 40 \\ 18 & 42 & \text { Lignières (Ldv. St. Johannsen) } & 94 \\ 41 & & & \end{array}$

dingte Staffelung der Versteigerungsdaten und -orte an den über weite Teile des Mittellandes zerstreuten Zehntbezirken des Stifts Bern zeigen: der jährliche Turnus begann in Oberwyl bei Büren für die am Jurafuß gelegenen Gebiete; dann folgte Radelfingen bei Aarberg für die Bezirke auf dem Frienisberger Plateau, Bern für diejenigen im Umkreis der Stadt, Oberbalm für die südwestlich angrenzende Hügelzone und zuletzt Rüeggisberg für das hochgelgene Einzelhof- und Weilergebiet des Längenbergs. Figur 2 stellt den mittleren Versteigerungstermin aller 42 Serien als Funktion der Höhe dar. Mit durchgezogenen Strichen ist der Höhenbereich angegeben, in welchem sich das Gros der von einer bestimmten Ortschaft aus versteigerten Zehntbezirke be- fanden. Auf vereinzelte Bezirke in größerer Höhenlage weisen die gestrichelten Linien hin. Die Figur veranschaulicht, daß die Versteigerungen jeweilen um den 10. Juli in den am tiesten gelegenen und phänologisch frühesten Bezirken um Brugg am «Wassertor der Schweiz» und am Unterlauf der Thur begannen und dann dem reifenden Getreide Schritt für Schritt in die Hügelzone des Höheren Mittellandes folgten, bis hinauf zu den Grenzertragsgebieten des Tessenbergs, des Schwarzenburgerlandes und des Emmentals in Höhenlagen von mehr als $800 \mathrm{~m}$, wo die Mitteldaten in die erste Augustdekade fallen. Die durchschnittliche zeitliche Differenz zwischen der tiefstgelegenen Landvogtei Schenkenberg (rund $350 \mathrm{~m}$ ) und den höchstgelegenen 
Räumen Schwarzenburg und Längenberg (Rüeggisberg, um $950 \mathrm{~m}$ ) beträgt 28 Tage, was der von volz (1978) am Beispiel der phänologischen Beobachtung der Weizenernten 1970-1974 errechneten Verzögerung von 4,6 Tage/100 m erstaunlich gut entspricht. Auch die vom selben Autor festgestellte relative Verspätung der Gebiete am Jurarand im Vergleich zu solchen in gleicher Höhe am Alpenrand scheint in den historischen Daten hervorzutreten, indem die Zehnten in den zwischen 800 und $900 \mathrm{~m}$ gelgenen Tessenberggemeinden später verliehen wurden als in den Gebieten um Rüeggisberg und Schwarzenburg (900-1000 m).

Die mit Dreiecken markierten Mittelwerte aus den direkten Beobachtungen (Tab. 1) schichten sich recht gut in das Bild der höhenabhängigen Verspätung der Versteigerungsdaten ein, vielleicht mit Ausnahme des Wertes von Gurzelen, der wohl durch die spätere Reife des Dinkels im Vergleich zum Roggen und die Häufung kühler Frühsommer während der Beobachtungsperiode bedingt ist (vgl. Fig. 3). Daneben erstaunt die weitgehende Übereinstimmung zwischen den Winterthurer Mittelwerten des 17 . und 18 . Jahrhunderts $(B, C)$ und demjenigen von Unter-Hallau aus dem 19./20. Jahrhundert (A). Wesentliche Differenzen zeigen sich dagegen im Ausmaß der Standardabweichung und der Schwankungsbreite (vgl. Tab. 1); im 17. Jahrhundert liegen zwischen dem frühesten Jahr und dem spätesten 35 Tage, gegenüber 27 Tagen in der Periode 1721-38 und 26 Tagen im 19./20. Jahrhundert.

\section{Die Homogenisierung}

Die Serien von Zehntsteigerungsdaten weisen entsprechend der fragmentarischen Natur historischer Quellen unterschiedliche Längen auf, sind lückenhaft und beziehen sich auf verschiedene Zeitabschnitte, Räume und Höhenstufen. Ferner galt in den protestantischen Kantonen Bern und Schaffhausen bis 1701 der julianische Kalender. Die Homogenisierung dieses Materials erwies sich als sehr zeit- und Computerkosten-aufwendig. Sie erfolgte in den folgenden fünf Schritten:

4.1. Umrechnung der Kalenderdaten in Datumszahlen. Dabei mußte auf Schaltjahre Rücksicht genommen und bei den Schaffhauser und Berner Daten für die Zeit vor 170110 Tage, für 170111 Tage hinzugezählt werden.

\subsection{Korrelation der einzelnen Serien unter sich sowie mit} den Monatstemperaturen (Februar bis Juli) der Basler Reihe (BIDER, SCHÜEPP, v. RUDLOFF 1959). Damit konnte ermittelt werden, welche Serien am besten zur Interpellation von «missing data» geeignet waren und welche von den weiteren Berechnungen ausgeklammert werden konnten. Unter sich waren die einzelnen Serien häufig mit Koeffizienten zwischen 0,6 und 0,85 bei einer Signifikanz von 0,001 korreliert. Viele mußten jedoch auf Grund ihrer ungenügenden Länge eliminiert werden. Die meisten Serien sind außerdem mit der Temperatur in einem oder mehreren Monaten zwischen April und Juli gut korreliert, am häufigsten mit den Mai- und Junitemperaturen (vgl. Tab. 2).

4.3. Berechnung von Mittelwerten für die Basisperiode 1755-1825. Bei den Schaffhauser Serien und denjenigen des Klosters Muri, die nicht oder nicht genügend weit in diese Periode hineinreichen (vgl. Tab. 2) mußten die entsprechenden Mittelwerte geschätzt werden. Dies geschah mit Hilfe von mehreren Serien, die sich mit den Schaffhauser und Kloster-Muri-Reihen über mehrere Jahrzehnte überlappten und gleichzeitig den ge-

Tabelle 2: Korrelation von Zehnsteigerungsdaten mit den Temperaturen (Februar bis Juli) in Basel

\begin{tabular}{llllrl}
\hline Periode & $\mathrm{r}$ & $\mathrm{S}$ & Standard-Error & \multicolumn{2}{c}{ Regressionsgleichung } \\
\hline Mai bis Juni & $-0,80$ & 0,00001 & 0,6 & $0,056-0,13676$ & $*$ ZD \\
Juni bis Juli & $-0,71$ & 0,00001 & 0,77 & $-0,11287-0,13139$ & $*$ ZD \\
Mai bis Juli & $-0,82$ & 0,00001 & 0,5 & $0,00393-0,12947$ & $*$ ZD \\
März bis Juli & $-0,86$ & 0,00001 & 0,46 & $-0,09424-0,12803$ & $*$ ZD
\end{tabular}

ZD: mittleres Residuum der Zehntsteigerungsdaten.

Die mittleren Residuen sind auf der Basis folgender Serien berechnet worden:

1611-1640: Mittel Andelfingen, Illnau, Winterthur (STBW 1) (vgl. Tab. 1)

1641-1662: Mittel Andelfingen und Illnau, (München)-Buchsee

1663-1679: Mittel Andelfingen und Illnau, (München)-Buchsee, Erlach, Tessenberg, Mittel der 5 Kloster Muri-Serien (vgl. S. 26)

1680-1697: Mittel Andelfingen und Illnau, (München)-Buchsee, Erlach, Tessenberg, Thorberg/Koppigen, Thorberg, Mittel der 5 Kloster Muri-Serien.

1698-1740: (München)-Buchsee, Erlach, Tessenberg, Thorberg/Koppigen, Thorberg, Fraubrunnen, Mittel der 5 Kloster Muri-Serien.

1741-1825: (München)-Buchsee, Erlach, Tessenberg, Thorberg/Koppigen, Thorberg, Fraubrunnen, Kirchberg (Kornamt), Landshut, Thun, Büren, Aarberg. 
samten Basiszeitraum 1755-1825 umfaßten. Die durchschnittliche Differenz der Mittelwerte zwischen Überlappungsperiode und Basisperiode wurde sinngemäß auf die älteren Reihen übertragen.

4.4. Ergänzung von Lücken in den verbleibenden Serien. Diese erfolgten durch Schätzwerte, welche anhand der Differenz der Mittelwerte berechnet wurden.

Aus den Regressionsgleichungen ergibt sich, da $ß$ ein Vorsprung oder Rückstand der Zehntverleihung von etwas mehrals 7 Tagen einer Abweichung derTemperaturum $1{ }^{\circ} \mathrm{C}$ vom Mittel der Monate Mai und Juni in der Basisperiode 1755-1825 entspricht. Ein ähnliches Resultat liefert die Analyse der phänologischen Reihe von Unter-Hallau (PFISTER, 1978 b). Anhand der letztzitierten Arbeit zeigt sich auch, daß säkulare Witterungsextreme die Gültigkeit des Modells in Frage stellen können: so täuscht der außergewöhnliche Vegetationsvorsprung des Jahres 1893 eine starke positive Abweichung der Mai-JuniTemperaturen vor, während die Extremtemperaturen in Wirklichkeit im April und Mai gemessen wurden. Deshalb empfiehlt sich in solchen Fällen ein Vergleich mit Beschreibungen des Witterungsverlaufs.

4.5. Berechnung der durchschnittlichen Residuen für die einzelnen Jahre. Von den verbleibenden Serien wurden die Mittelwerte der Basisperiode 1755-1825 subtrahiert und die Residuen für jedes Jahr ermittelt. Dabei standen jedoch nicht in jedem Zeitabschnitt gleichviele und auch nicht immer dieselben Serien zu Verfügung: zwischen 1611 und 1644 basieren die Werte nur auf den Reihen von Andelfingen und Illnau, und den doppelt gewichteten direkten Beobachtungen des Erntebeginns um Winterthur (Tab. 1). Zwischen 1641 und 1741 nimmt die Zahl der verwendeten Serien dann auf $11 \mathrm{zu}(\mathrm{vgl}$. Tab. 2). Dies wirkt sich dahingehend aus, daß die Einzelwerte und damit allfällige Abweichungen immer weniger ins Gewicht fallen, je weiter wir ins 18. Jahrhundert hineinkommen. Um den Einfluß systematischer Fehler zu verringern, wurden Residuen auf der Basis von geschätzten Mittelwerten stets weniger stark gewichtet als die übrigen.

\section{DieZehntsteigerungsdatenalsKlima-Indikatoren}

Die mittlere Abweichung der Zehntsteigerungsdaten aus verschiedenen Räumen und Höhenlagen vom Durchschnittswert der Basisperiode 1755-1825 wurde als Schätzgröße der Abweichung der Monatstemperaturen der Basler Reihe vom Mittelwert derselben Periode (unter Ausschluß der Jahre 1798-1802, für welche keine Zehntdaten vorliegen) gegenübergestellt. Es zeigte sich, daß der Einfluß der Temperatur auf den Zeitpunkt der Zehntsteigerung vom Vorfrühling bis zum Frühsommer von Monat zu Monat zunimmt, in den Monaten Mai ( $r=0,55)$ und Juni $(r=0,66)$ ein Maximum erreicht und dann im Juli wiederum schwächer wird. Vergleichen wir die 57 Beobachtungen der Roggenernte in Unter-Hallau SH aus der Zeit von 1888-1950 mit den Temperaturen und der Sonnenscheindauer mit den entsprechenden Monaten (PFISTER, 1978 b), erhalwir ein sehr ähnliches Bild:

erklärte Varianz $\left(\mathrm{R}^{2}\right)$ total: $\quad 59 \%$

davon Sonnenscheindauer Mai: $18 \%$

Temperatur Juni: $\quad 29 \%$

Sonnenscheindauer Juli: $\quad 12 \%$

An die Stelle der Temperatur tritt im Mai die Sonnenscheindauer, welche für den Zeitpunkt der Blüte von Bedeutung ist, aber im übrigen mit der Temperatur hoch korreliert ist. Bei der Sonnenscheindauer im Juli dürfte vor allem die erste Monatshälfte ausschlaggebend sein, in welcher in Unter-Hallau die Ernte stattzufinden pflegte (vgl. Tab. 1).

Infolgedessen wurde die Durchschnittstemperatur der Monate Mai und Juni als geeignetste Schätzgröße betrachtet.

Die Umrechnung der Schätzwerte von der Basisperiode 1755-1825 auf die Normalperiode 1901-60 erfolgte durch Subtraktion der durchschnittlichen Differenz der Mai-Juni-Temperatur $\left(0,2^{\circ}\right)$. (Die Einzelwerte jedes Jahres sind auf einem Datenträger gespeichert und werden im Rahmen einer «Klimageschichte der Schweiz» publiziert werden.)

\section{Getreide-Erntebeginn und Frühsommer- temperaturen seit dem 17. Jahrhundert}

In Fig. 3 sind auf der untersten Kurve für die Zeit von 1611-1825 die Residuen der Zehntsteigerungsdaten, von 1876-1950 die in Lohn SH und Unter-Hallau SH beobachteten Zeitpunkte des Getreide-Erntebeginns dargestellt. Die zweite Kurve zeigt bis 1754 die geschätzten, von 1755 an die in Basel gemessenen Temperaturen, immer in Form von Abweichungen vom Mittelwert 1901-60. Die dritte Kurve ist eine Darstellung der zweiten in Form von fünfjährig gleitenden Mitteln.

Erntedaten und Mai-Juni-Temperaturen werden nach folgenden 3 Gesichtspunkten untersucht:

1. Übereinstimmungen mit der langfristigen Temperaturentwicklung in anderen Räumen, insbesondere England und Nordfrankreich.

2. Übereinstimmungen mit den Schwankungen der Alpengletscher.

3. Unterschiede der Variabilität zwischen verschiedenen Zeitabschnitten.

\section{1. Übereinstimmungen mit der Temperaturentwicklung in Nordwesteuropa}

Der Vergleich kann sich für England von 1659 an auf die Temperaturreihe von MANLEY (1974) stützen. Für Nordfrankreich liegen die Weinlesedaten von LE ROY LADURIE (1971) vor. Beide Reihen verzeichnen recht befriedigende Übereinstimmungen mit den Zehntsteigerungsdaten: die Temperaturreihe korreliert auf einem Niveau von 0,68 (S. 0,0001, vgl. Fig. 4), die Weinlesedaten auf einen solchen von 0,64 (S. 0,0001). In den 
Fig. 3: Erntedaten und Temperaturen, 1611-1975



TEMPERATUREN MAI-JUNI (GLEITEND)
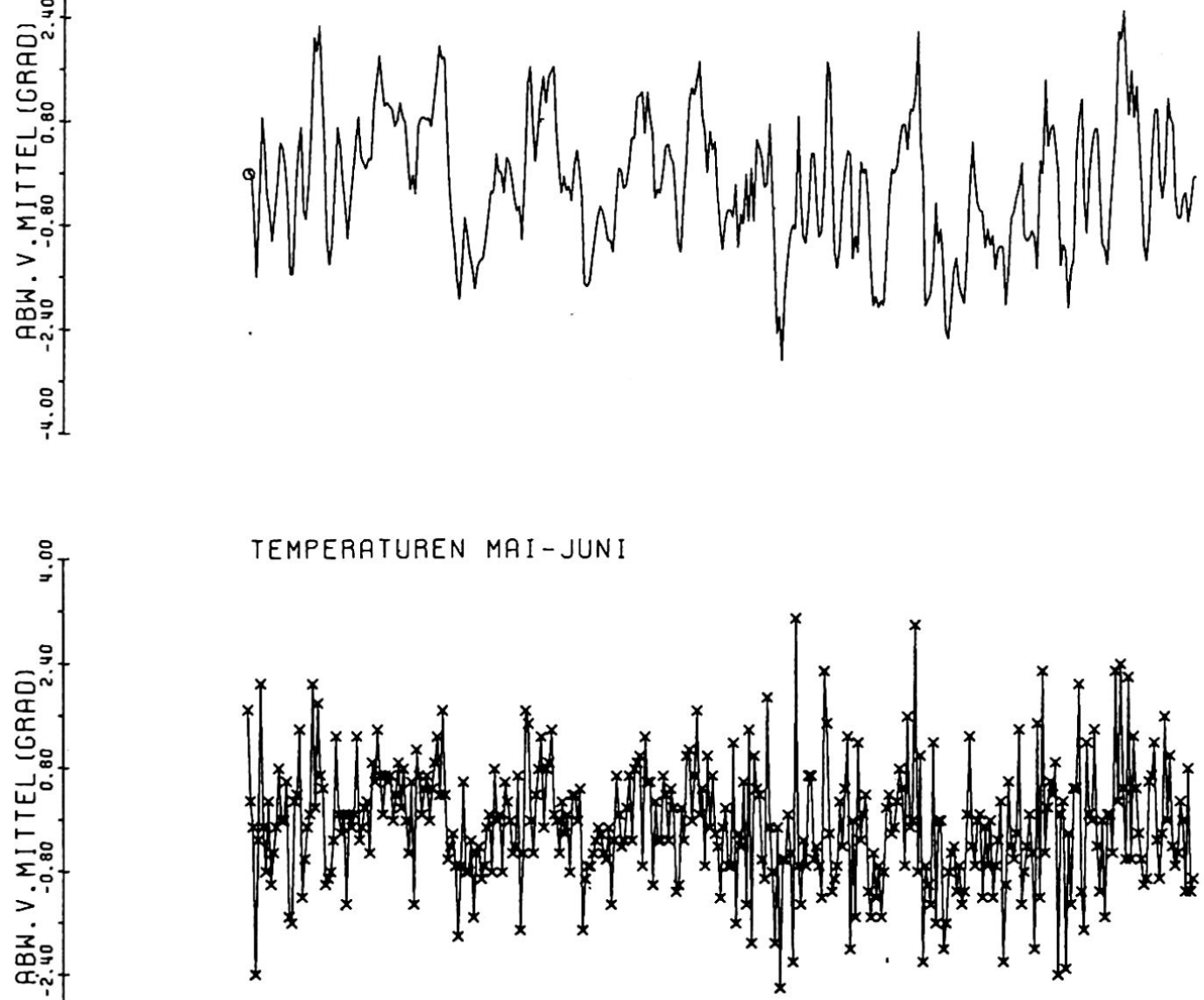

:

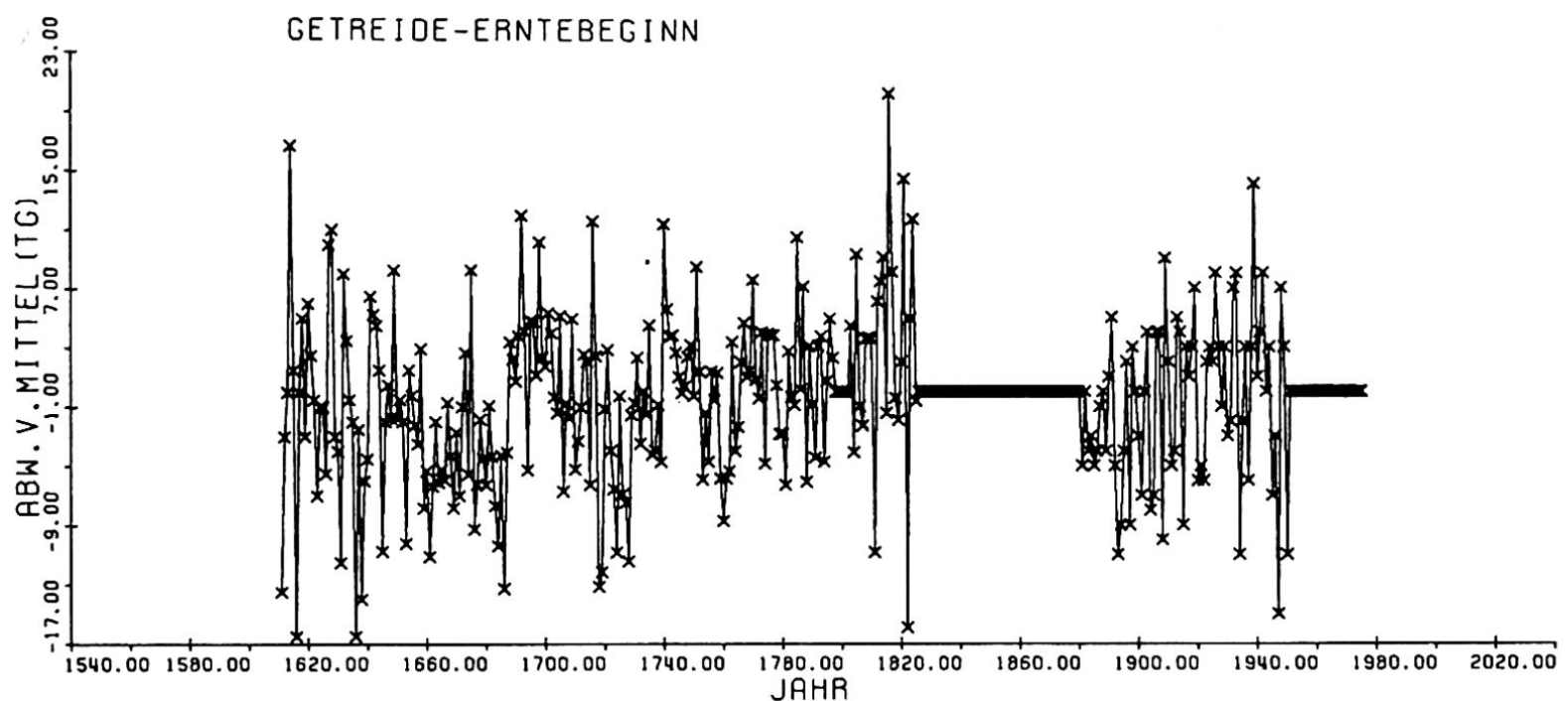


Fig. 4: Temperaturen und Niederschlagshäufigkeit im Mai-Juni und mittlere Erntedaten 1659-1759
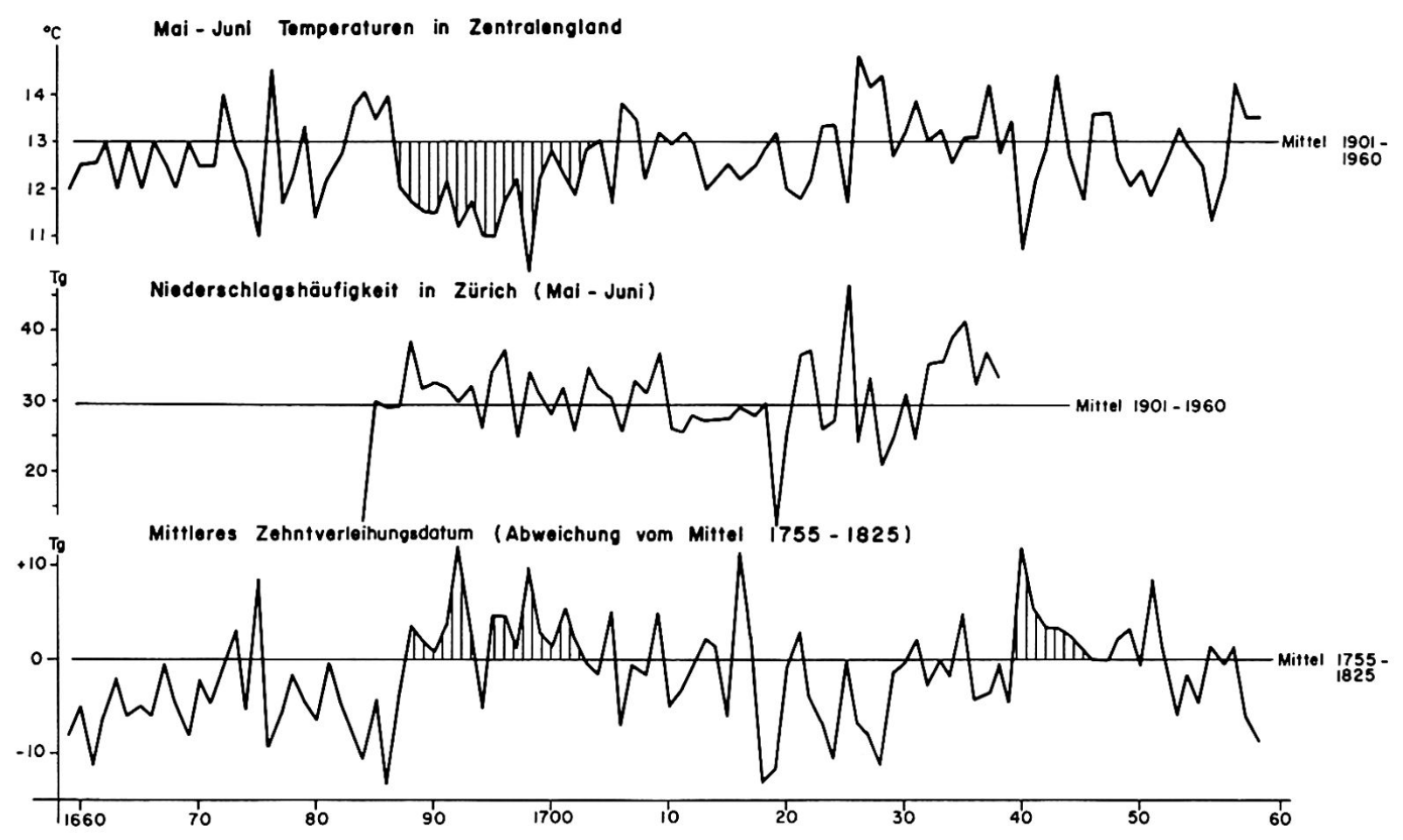

Quelle: Temperaturen in Zentralengland: MANLEY (1974)

Niederschlagshäufigkeit in Zürich: PFISTER (1978a)

beiden ersten Jahrzehnten (1611-1630) fehlt eine klare Tendenz; sie sind eher kühl, schließen aber extrem heiße Frühsommer wie diejenigen von 1611 und 1616 ein. In den 1630er Jahren, namentlich in der zweiten Hälfte, überwiegt die Erwärmung und setzt sich, abgesehen vom kühlen Intermezzo von 1641-44, für die nächsten zweieinhalb Jahrzehnte durch. Erst in der zweiten Hälfte der 1660er und den frühen 1670er Jahren ist der Trend wieder gegenläufig.

Der brüske Übergang von warmen zu kalten Frühsommern um 1687 signalisiert den Beginn eines der kältesten Abschnitte des «Little Ice Age»: für mehr als ein Jahrzehnt bleiben die Temperaturen in England um 1-1,5 $5^{\circ}$ unter den Mittelwerten des 20. Jahrhunderts, entsprechende Beträge wurden in anderen Jahreszeiten erreicht (MANLEY 1974). Von der Verspätung der Vegetationsentwicklung wurden Grenzertragsgebiete wie das schottische Hochland besonders hart getroffen. Der Hafer, die Grundnahrung der Unterschichten, kam nicht mehr zur Reife, weshalb die Sterblichkeit zwischen 1695 und 1700 höher lag als selbst zur Zeit des Schwarzen Todes (1348-50) (LAMB 1977). In der Schweiz fanden die Zehntverleihungen 8-10 Tage später statt als in den Jahrzehnten zuvor, was eine Temperaturdepression für Mai und Juni von nicht ganz einem Grad im Vergleich zu 1901-60 andeutet. Die tatsächliche Vegetationsverspätung dürfte größer gewesen sein. Von den Wintern wissen wir, daß der Schnee im Jahrzehnt 1691-1700 in Zürich im Durchschnitt fast doppelt so lang lag wie in der Zeit zwischen 1901 und 1960, was einer um 2 Grad tieferen Mitteltemperatur (Dezember bis März) entspricht (PFISTER 1978a). Die Erwärmung nach 1700 scheint, wie in England, alle Jahreszeiten erfaßt zu haben; sie gipfelt für die Frühsommertemperaturen in der Zeit zwischen 1718 und 1730 , in welche die vier ausgesprochen heißen und teilweise dürren Sommer 1718, 1719, 1724 und 1728 fallen. Doch währenddem die 1730er Jahre in England den Höhepunkt der sommerlichen Erwärmung bringen, ist die Tendenz zu kühleren und vor allem nasseren Sommern in der Schweiz unverkennbar (vgl. Fig. 4). Noch größer sind die Divergenzen in den 1740er Jahren, wo die drei in England ausgesprochenen warmen Frühsommer 1743, 1746 und 1747 in den Zehntsteigerungsdaten überhaupt nicht entsprechend in Erscheinung treten. Vom Ausmaß und der Wiederholungsneigung der frühsommerlichen Vegetationsverspätung her betrachtet, sind die Frühsommer der 1740er und frühen 1750er Jahre einzig mit dem Jahrzehnt 1690-1700 zu vergleichen. Ein entsprechendes Bild zeigen die nordfranzösischen Weinlesedaten (LE ROY LADURIE, 1971). Von 1753 an verlieren die Zehntsteigerungsdaten ihren Wert als Temperatur-Indikatoren, weil wir über homogenisierte Meßreihen verfügen (SCHÜEPP, 1960). Erwäh- 
nenswert ist höchstens, daß die Zehntdaten die kühlere Periode von 1766-1777 wesentlich deutlicher nachzeichnen als diejenigen von 1812-1817.

\subsection{Die Übereinstimmung mit Schwankungen von Alpengletschern}

Zu den wichtigsten Klimaparametern, von welchen die Massenbilanz eines Gletschers abhängt, rechnet man die Häufigkeit und Verteilung der Neuschneefälle, die Temperatur und Strahlung während der Ablationsperiode (FLIRI, 1964, HOINKES, 1967). VIVIAN (1975) verglich in einer Modellrechnung die monatlichen Vorstoßbeträge des «mer de glace» mit den Temperaturen und kam zum Schluß, daß die Temperatur im Juni gegenüber den übrigen Monaten für das Ausmaß der Zungenlängenänderung wesentlich stärker ins Gewicht fällt; dieses Resultat ist in unserem Zusammenhang insofern interessant, als die Juni-Temperaturen auf die Reife des Wintergetreides ebenfalls besonders staken Einfluß haben.

Ein Vergleich von Gletscherschwankungen und Zehntsteigerungsdaten wird bis zur Mitte des 18. Jahrhunderts durch die verhältnismäßig spärliche Dokumentation über das Gletscherverhalten erschwert. Die vorhandenen schriftlichen Aufzeichnungen und Bilddokumente erlauben nur in wenigen Fällen eine quantitative Abschätzung des Gletscherstandes. LE ROY LADURIE (1971) und ZUMBÜHL (1976) stimmen dahingehend überein, daß der erste größere Rückzug der alpinen Talgletscher von den Maximalständen des frühen 17. Jahrhunderts in die Zeit zwischen 1640 und 1680 fällt. ZUMBÜHL (1976) deutet in seiner Graphik an, daß der Untere Grindelwaldgletscher in der Zeit von 1640 bis 1686 um einen Betrag von 400 bis $800 \mathrm{~m}$ zurückschmolz und möglicherweise in der Mitte der 1680er Jahre einen während der Phase des "Little Ice Age» nie wieder egalisierten Minimalstand erreicht hatte. Zeitlich paßt dieser Befund in die durch die Zehntdaten signalisierten Warmphasen 1635-40, 1644-62 und 1679-84 (Figur 3).

In den folgenden Jahrzehnten will das Verhalten der Alpengletscher nicht mehr in das klimatische Szenario, kühl-feuchte Sommer und kalte, schneereiche Winter, passen. Nach LE ROY LADURIE (1971) schmelzen viele Gletscher im Jahrzehnt 1690-1700 weiter zurück, während die Graphik von ZUMBÜHL (1976) für den Unteren Grindelwaldgletscher eine Trendumkehr um 1690 und eine anschließende Vorstoßphase andeutet. Beide Autoren signalisieren Vorstöße im frühen 18. Jahrhundert mit einem relativen Maximum um 1720, was bedeutet, daß die Expansion der Gletscherzungen zeitlich mit einer Erwärmung der Frühsommer und der Winter einherging. Die anschließende Rückzugsphase kann klar den trocken-heißen Sommern der 1720 er Jahre zugeordnet werden und eine zunehmende Vorstoßneigung in den 1730er Jahren erscheint von den kühl-feuchten Frühsommern her ebenfalls plausibel. Größere Diskrepanzen zwischen dem Verhalten der Grindelwaldgletscher und den Zehntsteigerungsdaten ergeben sich in den 1740er Jahren: ZUMBÜHL (1976) signalisiert ein rasches Rückschmelzen des Unteren Gletschers nach 1743, während dem die späten Zehntverleihungen und Weinlesedaten (LE ROY LADURIE, 1978) für kühle Frühsommer sprechen. Ob der Untere Grindelwaldgletscher in diesen Jahrzehnten ein atypisches Verhalten zeigt oder ob Zehntsteigerungsund Weinlesedaten die thermischen Trends dieser Jahre unvollständig wiedergeben, müßte anhand von Angaben über das Verhalten weiterer Gletscher abgeklärt werden. Diskrepanzen in dieser Größenordnung sind jedenfalls in den zwei folgenden sowohl von der Witterung als auch vom Gletscherverhalten her besser dokumentierten Jahrhunderten nicht mehr festzustellen (MESSERLI et. al, 1976).

\subsection{Die Variabilität von Erntedaten und Temperaturen}

LAMB (1977) bezeichnet die größere Variabilität (Verhältnis von Standardabweichung und Mittelwert) der Temperatur von Jahreszeit zu Jahreszeit, von Jahr zu Jahr und zwischen Gruppen von ähnlichen Jahren als eines der charakteristischen Merkmale des «Little Ice Age». Es soll geprüft werden, inwieweit diese Tendenz im vorliegenden Datenmaterial zu Tage tritt.

Die dreißigjährige Periode zwischen 1611 und 1640 ist durch außergewöhnlich starke und häufige thermische Schwankungen gekennzeichnet. Im Jahre 1616 war der Roggen in Winterthur am 23. Juni schnittreif, eine Woche vor dem Erntebeginn des Jahres 1893, welcher in Unter-Hallau der früheste zwischen 1881 und 1950 war. Nur wenig später als 1616 begann die Ernte in den Jahren 1611, 1631, 1636 und 1638. Ein Vegetationsvorsprung in dieser Größenordnung deutet auf einen Wärmeüberschuß hin, welche demjenigen in den wärmsten Frühjahren und Frühsommern der letzten hundert Jahre $(1893,1934,1947,1950,1976)$ entsprach. Auf der anderen Seite weisen die Ernten der Jahre 1614, 1627 und 1628 einen Rückstand auf, der sich mit demjenigen in den Jahren 1879, 1898 und 1939 vergleichen läßt, wo ein frühsommerliches Wärmedefizit von 1,5 Grad gemessen wurde.

Insgesamt dürften 6-8 der 30 Jahre zwischen 1611 und 1640 Wärmeüberschüsse oder -defizite gebracht haben, die bezogen auf die Periode 1901-60, oberhalb des 11 . oder unterhalb des 1. Duodezils liegen. (Beim 1. und 11. Duodezil handelt es sich um Schwellenwerte, die von je $8 \%$ aller Meßwerte unter-bzw. überschritten werden.) Das heißt, die Zahl der Extremfälle dürfte fast doppelt so großgewesen sein wie in unserem Jahrhundert, worauf auch die im Vergleich zu 1880-1950 bei identischen Mittelwerten entsprechend größere Standardabweichung hindeutet (vgl. Tab. 1). Eine große Variabilität finden wir auch im Zeitraum 1711-1740, wobei die heißen und zum Teil dürren Frühsommer (1718, 1719, 1724, $1728)$ gegenüber den extrem kalten $(1716,1740)$ deutlich überwiegen. Die Standardabweichung der phänologischen Beobachtungen ist etwas kleiner als im frühen 17., 
aber noch deutlich größer als im 20. Jahrhundert (vgl. Tab.1).

Eine weitere Periode mit einer Häufung von Extremwerten und einer entsprechend überdurchschnittlichen Variabilität ist diejenige von 1803-25. Sie schließt das «Jahr ohne Sommer» (1816) ein, in welchem der Rückstand der Ernte den Rekordbetrag von drei Wochen erreichte. Das heißt, auch in den frühesten Lagen wurde vor Anfang August keine Sichel ins Feld geführt, und in den höheren Niveaus des Längenbergs auf 900 bis $1000 \mathrm{~m}$ begann die Ernte des Wintergetreides im September. Sommergetreide und Kartoffeln kamen auf dieser Höhenstufe nicht mehr zur Reife und wurden unter dem Ende Oktober fallenden Schnee begraben (PFISTER, 1975). Die große Hungersnot des folgenden Jahres, die letzte in der Schweiz, kostete Gebirgskantone wie Appenzell bis zu 10\% ihrer Bevölkerung (sCHÜRMANN, 1974). Eine Vegetationsverspätung in ähnlichem Ausmaß, vermutlich mit ähnlichen demographischen Konsequenzen, trat 1573 ein, ein Jahr, in welchem sich klimatische Extreme in allen Jahreszeiten in einer einzigartigen Weise häuften (PFISTER, in Vorb.). In der Periode 1803-25 finden wir den größten Sprung30 Tage - zwischen zwei aufeinanderfolgenden Jahren (1821, 1822), welcher einer Temperaturdifferenz von mehr als $4 \mathrm{Grad}$ entspricht.

Das «Little Ice Age», so können wir feststellen, zeichnet sich auch bezüglich der Mai-Juni-Temperaturen durch eine wesentlich größere Variabilität aus als die erste Hälfte des 20. Jahunderts.

Werfen wir abschließend einen Blick auf die meteorologischen Ursachen und die wirtschaftlichen Konsequenzen dieser Erscheinung: Der wohl beste Kenner der Klimageschichte, HUBERT LAMB (1977), zeigt auf, daß die größere Variabilität der Temperatur einer größeseren Häufigkeit von blockierenden Hochdruckgebieten am Rande des Kontinents und einer meridionalen, von Norden nach Süden gerichteten Zirkulationsform entspringt. Bei einer solchen Druckverteilung hat das Wetter die Tendenz, sich über Monate, manchmal über ganze Jahreszeiten hinweg festzufahren und seinen Charakter kaum zu ändern, wie dies in Nordwest- und Zentraleuropa zuletzt im Dürresommer von 1976 der Fall war. Je nachdem, ob ein Gebiet dann auf der linken «warmen» oder auf der rechten «kalten» Flanke des Hochdruckrückens liegt, ist der betreffende Zeitabschnitt deutlich zu warm oder zu kalt. Besonders ausgeprägt finden wir die Erscheinung im Jahre 1616: während man im Januar in Bern auf der gefrorenen Aare vom Marzili zum Sulgenbach lustwandelte und Kurzweil trieb, folgte im Juni eine beispiellose Hitzewelle. «Es hat auch von obgemelten großen Regen (am 6.Juni) nie mer geregnet bis auff den 30 . Julij, ist ein sanfter regen angestanden und der himmel alls mit Wolcken uberzogen worden, daß ein iegklich vermeint, ein langwiriges und nasses wetter zu erfolgen, hett doch nur 3 tag gewert ohnaufhörlich und ist das Embdt, daß zuvor verdörret gewesen, wider in ein junges greßli geschossen", notierte Abt Placidus Brunschwiler von Fischingeit
(TG) in sein Tagebuch (EINSD). Das bedeutet, daß die Trockenperiode (darunter versteht man eine Serie von mindestens 5 Tagen mit weniger als $0,3 \mathrm{~mm}$ Niederschlag) des Jahres 1616 in der Nordschweiz mehr als 50 Tage gedauert hat, rund doppelt so lang wie diejenige des heißen Sommers 1976, länger als die längste sommerliche Trockenperiode, welche seit Beginn der Messungen in diesem Gebiet verzeichnet worden ist (49 Tage vom 2. 7. bis 19.8.1911 in Kaiserstuhl AG) (COURVOISIER, MAEDER, PRIMAULT, 1976). Die Tatsache, daß der Winter 1616 in England zu den kältesten gehört (mdl. Mitt. H. H. LAMB) und die französischen Weinlesedaten in diesem Jahr einen im gesamten 17. und 18. Jahrhundert nicht mehr egalisierten Vorsprung von 23 Tagen verzeichnen (LE ROY LADURIE, 1971), legt dieVermutung nahe, daß die Verhältnisse in größeren Räumen Nordwest- und Mitteleuropas ähnlich waren.

Wenn das blockierende Hoch sich mehr am Rande des Kontinents aufbaut, rückt Mitteleuropa in den Einflußbereich einer Nordwestströmung, die frische Polarluft gegen die Alpen heranführt. Auch diese Wetterlage mit kaltem Regen in den Niederungen und Schnee in den Bergen - kann über Wochen und Monate hinweg stationär bleiben, wie dies zuletzt im Frühsommer 1978 der Fall war. Noch länger dürfte sich die Nordwestlage in den kalten und nassen Sommern 1627, 1628, 1673, $1675,1740,1770$ und vor allem 1816 erhalten haben. Die größten negativen Temperaturabweichungen ergeben sich unter dem Einfluß von blockierenden Hochdruckgebieten im Winter und Frühjahr. So verzeichnete der März 1785, für welchen KINGTON (1978) tägliche Wetterkarten rekonstruiert hat, im schweizerischen Mittelland eine ununterbrochene Schneebedeckung und ein Temperaturdefizit von fast $8 \mathrm{Grad}$, was im 20. Jahrhundert noch nie, auch nicht annähernd, der Fall gewesen ist (BIDER, SCHÜEPP, v. RUDLOFF, 1959; PFISTER, 1975). Wenn wir das Wetter nach seinen Auswirkungen auf die Wirtschaft beurteilen, steht die Frage nach der Variabilität im Vordergrund. Die Untersuchung über die Beziehung zwischen dem Klima und den landwirtschaftlichen Erträgen von JEANNERET (1978) hat ergeben, daß die Ertragsgestaltung landwirtschaftlicher Kulturpflanzen durch ein System von agroklimatischen Schwellenwerten gesteuert wird. Und zwar finden wir bei jedem Klimaparameter einen oberen und einen unteren Schwellenwert, dessen Überschreitung beziehungsweise Unterschreitung eine Beeinträchtigung des Ertrages bedeutet. Eine Persistenz desselben Witterungstyps, sei es in Form einer Trockenperiode, einer Zeit ausgedehnter Regenfälle und Überschwemmungen, einer Kältewelle oder anhaltender Schneefälle trifft neben der Landwirtschaft eine Vielzahl weiterer Wirtschaftszweige und vermag, wie das Beispiel der jüngsten Winter in den USA oder der Sommer 1976 und 1978 in Europa zeigt, den Lebensrhythmus ganzer Nationen empfindlich zu stören. Solche meteorologische Streß-Situationen sind in der Phase des sogenannten "Little Ice Age» häufiger aufgetreten als in der ersten Hälfte unseres Jahrhunderts. 
Wenn wir uns in eine Klimaphase hineinbewegen sollten, welche dem «Little Ice Age» ähnlich ist, worauf mehrere Indizien wie die Ausdehnung des Packeisgürtels, die markante Abkühlung im Polargebiet und die auflebenden Vorstöße von Alpengletschern hindeuten, dann wären die größten Veränderungen jedenfalls nicht bei den Mittelwerten, sondern im Ausmaß der Variabilitäten zu erwarten.

\section{Summary}

For the period between the early 17th and the early 19th century historical documents yield the dates, on which the tithes paid in grain were sold by auction. It can be shown that these dates were carefully chosen according to the stage of maturity of the crop and that maturity was essentially a function of the temperatures in May and June. The latter are estimated for the time since 1611 on the basis of a series combined from tithe-dates and phenological observations of the beginning of the harvest. The argument of a greater variability of the temperatures during the «Little Ice Age» brought forward by LAMB can be supported, especially for the early 17th and the early 18th century. Series of cold springs and early summers stand out from 1688-1702, 1740-51, 1766-71 and 1812-17. While the latter two cold periods coincide clearly with times of glacier advances, the glaciers seem to be out of phase during the earlier two periods.

Der Verfasser ist den folgenden Persönlichkeiten und Institutionen zu Dank verpflichtet:

- Proff. B. Messerli und M. Schüepp für das Durchlesen des Manuskripts und wertvolle Anregungen

- Climatic Research Unit, University of East Anglia Norwich U.K.

Geographisches Institut der Universität Bern

Meteorologische Zentralanstalt Zürich

Staatsarchive Aarau, Bern und Schaffhausen, Stiftsarchiv Einsiedeln.

\section{Literatur}

BIDER, M.; SCHÜEPP, M.; von RUDLOFF, H., 1958: Die Reduktion der 200jährigen Basler Temperaturreihe. Archiv für Meteorologie, Geophysik und Bioklimatologie. Serie B. 9: 360-412.

COURVOISIER, H.; MÄDER, F.; PRIMAULT, B., 1976: Die Dürre 1976 und ihre Auswirkungen. Arb. ber. der Schweiz. Meteorol. Zentralanst. Nr. 73, Zürich.

DANSGAARD, W.; JOHNSEN, S. J.; MÖLLER, J.; LANGWAY, C. C., 1969: One thousand centuries of climatic record from Camp Century on the Greenland ice sheet, Science, Vol. 166.

DE VRIES, J., 1977: Histoire du Climat et Economie. Des faits nouveaux, une interprétation différente. Annales E. S. C., 32: 198-226.
DICKEL, H., 1972: Der Beginn der Mähdreschernte des Roggens als phänologische Phase. Wetter und Leben, Jg. 24, Wien, S. 74-78.

DUFOUR, M. L., 1870: Problème de la variation du climat. Bull. Soc. Vaud. Sci. nat., Bd. 10, H. 63: 359-556.

EINSD: Stiftsbibliothek Einsiedeln. MF 25. Brunschwiler, Placidus, Diarium Fischingense (1616-1654).

FLIRI, F., 1964: Zur Witterungsklimatologie sommerlicher Schneefälle in den Alpen. Wetter und Leben, Bd. 16: 1-11.

FLOHN, H., 1949: Klima und Witterungsablauf in Zürich im 16. Jahrhundert. Vjschr. d. Naturf. Ges. Zürich, 94 :

28-41.

GMÜR, R., 1954: Der Zehnt im Alten Bern. Abh. zum schweiz. Recht, N. F., Bern.

HOINKES, H., 1967: Gletscherschwankung und Wetter in den Alpen. 9. Internat. Tagung f. alpine Meteorologie 14.-17.9.1966, Zürich: 9-24.

JEANNERET, F., 1978: Die Klimaeignung für die Landwirtschaft - Methode und Probleme einer gesamtschweizerischen Grundlagenkartierung (mit Kartenbeilagen). Geographica Helvetica, Bd. 33, Nr. 1: 49-54.

KOBLET, R., 1965: Der landwirtschaftliche Pflanzenbau unter besonderer Berücksichtigung der schweizerischen Verhältnisse. Basel.

LAMB, H. H., 1977: Climate. Present, Past and Future. Vol 2. Climatic History and the Future, London.

LE ROY LADURIE, E., 1971: Times of Feast. Times of Famine. A history of climate since the year 1000. London.

LE ROY LADURIE, E., 1978: Une synthèse provisoire: les vendanges du XVe au XIXe siècle. Annales E.S.C., H. 4 , im Druck.

MANLEY, G., 1974: Central England temperatures: monthly means 1659 to 1973 . Quart. J. R. Met. Soc. 100: 389-405.

MARGOLIS, S. V., KENNETT, J. P., 1971: Cenozoic paleoglacial history of Antarctica recorded in deep sea cores. Amer. J. Sci,. Bd. 271: 1-36.

MESSERLI, B. et al., 1976: Die Schwankungen des Unteren Grindelwaldgletschers seit dem Mittelalter. Ein interdisziplinärer Beitrag zur Klimageschichte. Ztschr. f. Gletscherkde. Bd. 11: 3-110.

PEYER, E. KOBLET, w., 1966: Der Einfluß der Temperaturund der Sonnenstunden auf den Blütezeitpunkt der Reben. Schweiz. Zeitschrift für Obst- und Weinbau. Vol. 102: 250-255.

PFISTER, Ch., 1975: Agrarkonjunktur und Witterungsverlauf im westlichen Schweizer Mittelland. Geographica Bernensia G 2. Bern.

PFISTER, Ch., 1978 a: Zum Klima des Raumes Zürich im späten 17. und frühen 18. Jahrhundert. Vierteljschr. natf. Ges. Zürich, Bd. 122, H. 4: 447-471.

PFISTER, Ch., 1978 b: Long phenological time series from the Canton of Schaffhausen (Switzerland) and their application for the interpretation of Historical Records. 
Climatic Unit Research Publications CRURP, Univ. of East Anglia, Norwich U. K., in print.

RÖTHLISBER GER,F., 1976: Gletscher-und Klimaschwankungen im Raum Zermatt, Ferpècle und Arolla. 8000 Jahre Walliser Gletschergeschichte. Die Alpen, Bd. 52, H. 3/4: 58-152.

SCHÜEPP, M., 1960, 1966: Lufttemperatur. 2., 3. und 4. Teil. Klimatologie der Schweiz C. Beiheft zu den Annalen der Schweiz. Met. Zentralanst.

SCHÜRMANN, M., 1974: Bevölkerung, Wirtschaft und Gesellschaft in Appenzell-Innerrhoden im 18. und frühen 19. Jahrhundert. Innerrhoder Geschichtsfreund. Appenzell.

SCHWEINGRUBER, F. H.: Dendroklimatische Studien an Nadelhölzern von Mittel- und Nordwest-Europa mit röntgenanlytischen Methoden. Vorauss. in Boreas.

SPJELDNESS, N.,1978: Climatic Changes in the Paleozoic. Proceedings of the Nordic Symposium on Climatic Changes and related Problems, Copenhagen 24.-28. April 1978: 76-84.

STAUFER, B. und LÜTHI, A., 1975: Wirtschaftsgeschichtliche Quellen im Dienste der Klimaforschung. Geographica Helvetica, Bd. 30: 49-56.

STAB 1: Staatsarchiv Bern: B VI 153. Brief des Landvogts S. A. Steiger von Bipp vom 8.8.1770.

STAB 2: Staatsarchiv Bern: B VI 184. Brief des Oberamtm. von Wangen vom 15.7.1806.

ST AB 3: Staatsarchiv Bern: Urbarien Erlach, Nr.101. Brief der Dt. Seckelschreiberei vom 9.3.1752.

STAB 4: Staatsarchiv Bern. Zehntrödel.

(München)-Buchsee 1638-1797. Urbarien Amt Fraubrunnen, Nr. 146-149.

Erlach/Tessenberg 1663-1825. Urbarien Erlach, Nr. 95 bis 101.

Koppigen/Thorberg 1680-1704. Urbarien Amt Burgdorf, Nr. 43.
Fraubrunnen 1698-1825. Urbarien Amt Fraubrunnen, Nr. 147, 148.

Wangen 1727-1746. B VI 561.

alle deutschen Vogteien 1741-1797. B VI 124-180.

neuer Kanton 1803-1825. B VI 181-202.

STAG: Staatsarchiv Aargau. Nr. 5931-5935. Kloster Muri Zehntrödel. 17. und 18. Jahrhundert.

STASH: Staatsarchiv Schaffhausen. BA 26, Kloster Allerheiligen Zehntrödel, 17. und 18. Jahrhundert.

STBW 1: Stadtbibliothek Winterthur. Ms. Q 72: Künzli, A., Chronik (ausführlich von 1611 bis 1644).

STBW 2: Stadtbibliothek Winterthur. Ms. Q 238 1-16. Rieter, H. R., Verzeichnis über das Jahr . . . (1721-1738) 16 Bde.

TRENKLE H., 1969: Die Verwendung phänologisch-klimatologischer Beobachtungen bei der Gütebewertung von Weinbergslagen. Die Weinwissenschaft. Vol. 24: 327-338.

VIVIAN, R., 1975: Les glaciers des Alpes Occidentales. Grenoble.

volZ, R., 1978: Phänologische Karte von Frühling, Sommer und Herbst als Hilfsmittel für eine klimatische Gliederung des Kantons Bern. Jahrbuch der Geographischen Gesellschaft Bern 52 (1975/6).

wolf, R., 1853: Jahr Rodel von Hans und Abraham Wieniger, Schulmeistern von Bedderkinden 1716-1770. Notizen z. Gesch. der Math. und Phys. in der Schweiz. 30. Mitt. natf. Ges. Bern, Bd. 7: 140-152.

ZUMBÜHL H. J., 1976: Die Schwankungen des Unteren Grindelwaldgletschers in den historischen Bild- und Schriftquellen des 12. bis 19.Jahrhunderts.

in: MESSERLI, B. et al.: Die Schwankungen des Unteren Grindelwaldgletscher seit dem Mittelalter. Ein interdisziplinärer Beitrag zur Klimageschichte. Ztschr. f. Gletscherkde Bd. 11, H. 1: 12-50.

\section{Literaturbesprechungen}

LAUER, Wilhelm/KLINK, Hans-Jürgen (Hrsg.) : Pflanzengeographie, Wege der Forschung CXXX, div. Beiträge, 573 S., Wissenschaftliche Buchgesel1schaft, Darmstadt, 1978, ISBN 3-534-04802-4. DM 85,- (Mitglieder DM 57,-)

Es war einmal selbstverständlich, dass sich ein Geograph auch in der Botanik auskannte. So erbrachten die zahlreichen geographischen Forschungsreisen stets auch ein reiches pflanzenkundliches Material. Mit Carl Troll, dem der besprochene Band gewidmet ist, endet die Reihe der klassischen Pflanzengeographen. Dennoch sind die diplomatiscleen Beziehungen zwischen Geographie und Botanik nicht abgebrochen. Ueber den modernen Begriff der Oekologie sind die beiden Disziplinen erneut in engen Kontakt getreten. Die Erkenntnisse der Geobotaniker kommen wieder zu Ehren und werden durch neue Betrachtungsweisen ergänzt. Darum gibt die hier gebotene Zusammenstellung der wesentlichsten Beiträge zu diesem Fachgebiet von Grisebach bis Ellenberg eine gute Grundlage und weckt hoffentlich das vermehrte Interesse der Geographen für diese Wissenschaft. 